\title{
ATLAS Measurements of Jet Suppression in Heavy Ion Collisions
}

\author{
Aaron Angerami on behalf of the ATLAS Collaboration
}

Columbia University, Nevis Labs, Irvington, NY, 10533, USA

\begin{abstract}
The first results of single jet observables in $\mathrm{Pb}+\mathrm{Pb}$ collisions at $\sqrt{s_{\mathrm{NN}}}=2.76 \mathrm{TeV}$ measured with the ATLAS detector at the LHC are presented. Full jets are reconstructed with the anti- $k_{\mathrm{t}}$ algorithm with $\mathrm{R}=0.2$ and 0.4 , using an event-by-event subtraction procedure to account for the contribution of the underlying event (including elliptic flow) to the reconstructed jet energy. The geometrically-scaled ratio of jet yields in central and peripheral events, $R_{\mathrm{CP}}$, indicates a clear suppression of jets with $E_{\mathrm{T}}>100 \mathrm{GeV}$. No significant change in the level of suppression is found when moving from $\mathrm{R}=0.2$ to $\mathrm{R}=0.4$.

Keywords: Heavy ion, jets, jet quenching

PACS: $25.75 .-\mathrm{q}$
\end{abstract}

\section{INTRODUCTION}

Energetic jets produced in high energy nuclear collisions serve as probes of the hot, dense medium created there by the phenomenon of "jet quenching" [1]. This phenomenon was investigated by the RHIC experiments, particularly through the measurement of the $R_{\mathrm{AA}}$ of single hadrons [2,3]. The observed violation of binary scaling indicates a breakdown of factorization in heavy ion collisions [1]. Despite this insight, the published RHIC measurements have been limited to using single particles as a proxy for the entire jet, thus providing only indirect evidence for jet quenching. To draw further conclusions requires full jet reconstruction.

Dijet pairs, where each jet traverses a different length of plasma, should be sensitive to energy loss, especially in the extreme scenario where one jet is entirely extinguished by the medium [4]. The ATLAS measurement of dijet asymmetry experimentally addresses this possibility [5]. This observation of modification of the dijet asymmetry in $\mathrm{Pb}+\mathrm{Pb}$ collisions relative to $\mathrm{p}+\mathrm{p}$ was the first published measurement of fully reconstructed jets in heavy ion collisions. By itself, the result is strongly suggestive of jet quenching. However, to definitively prove the quenching of jets further measurements are needed.

Single jet production rates are expected to be suppressed by jet quenching. Calculations of radiative energy loss predict a dependence of the measured suppression on the radius of the jet in the $\eta-\phi$ plane [6]. If jet quenching causes the jet's energy to be distributed to larger angles, the suppression in the jet rate could be reduced by enlarging the radius in the jet definition, recovering more of the jet's energy. In the absence of a measured $p+p$ spectrum, the central to peripheral ratio, $R_{\mathrm{CP}}$, can be used:

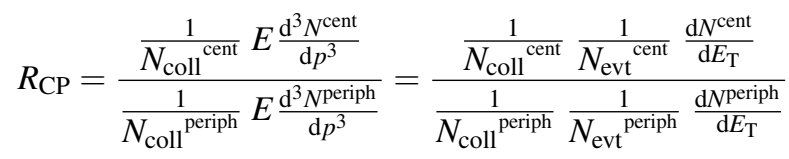

\section{JET RECONSTRUCTION}

The most important features of the analysis procedure are presented here and closely follow Ref. [7]. A detailed account of the analysis can be found in Ref. [8]. The jet reconstruction procedure in heavy ion collisions assumes that the energy of the jet is superimposed on the background from the underlying event:

$$
\frac{\mathrm{d}^{2} E_{\mathrm{T}}^{\text {tot }}}{\mathrm{d} \eta \mathrm{d} \phi}=\frac{\mathrm{d}^{2} E_{\mathrm{T}}^{b k g r}}{\mathrm{~d} \eta \mathrm{d} \phi}+\frac{\mathrm{d}^{2} E_{\mathrm{T}}^{j e t}}{\mathrm{~d} \eta \mathrm{d} \phi} .
$$


Since the background is not known it must be estimated from the rest of the event. This is achieved by considering the $\eta$-dependent average background level in regions unbiased by jets and the $E_{\mathrm{T}}$ modulation due to elliptic flow.

$$
\frac{\mathrm{d}^{2} E_{\mathrm{T}}^{b k g r}}{\mathrm{~d} \eta \mathrm{d} \phi} \sim\left\langle\frac{\mathrm{d}^{2} E_{\mathrm{T}}}{\mathrm{d} \eta \mathrm{d} \phi}\right\rangle\left[1+2 v_{2} \cos \left(2\left(\phi-\Psi_{2}\right)\right)\right]
$$

The measurements of jet observables that follow use this background estimation procedure applied to Eqn. 2 to correct the energies of jets reconstructed with the anti- $k_{\mathrm{t}}$ algorithm [9]. This algorithm is infrared-safe to all orders and produces cone-like jets that are geometrically well-defined with a size controlled by a parameter $R$. Reported here are measurements with both $\mathrm{R}=0.2$ and $\mathrm{R}=0.4$. Calorimeter towers of size $\Delta \eta \times \Delta \phi=0.1 \times 0.1$ are used as inputs to the reconstruction. Each tower is composed of several longitudinal sampling layers weighted using energy-density dependent factors to correct for calorimeter non-compensation and other energy losses [10]. An overall, multiplicative energy scale correction is applied to each reconstructed jet [11].

The raw spectra are corrected for resolution and residual jet energy scale errors by using a bin-by-bin unfolding procedure based on Monte Carlo studies. Samples of PYTHIA p+p jet events and HIJING $\mathrm{Pb}+\mathrm{Pb}$ events were generated and run through a full GEANT simulation of the ATLAS detector. They were then merged into a single event and reconstructed in an identical fashion to the data. Per-bin correction factors were extracted from these samples and used to correct the data. Variation of the correction factors as well as the jet energy resolution and centrality variation of the jet energy scale are included in the systematic uncertainties indicated by the grey shaded regions in the figures.

\section{RESULTS}

Data recorded by ATLAS during the 2010 lead ion run is used in this analysis corresponding to a total integrated luminosity of $7 \mu \mathrm{b}^{-1}$. Events are selected using minimum bias event criteria chosen to select in-time collisions and reject non-collision and photo-nuclear backgrounds, resulting in a data set containing 47 million events. These events are partitioned into centrality classes based the amount of transverse energy deposited in the forward calorimeter $(3.2<|\eta|<4.9)$. In this analysis, jets are restricted to the interval $|\eta|<2.8$.

The $R_{\mathrm{CP}}$ is shown in Fig. 1 for the jets using $\mathrm{R}=0.2$ and in Fig. 2 for the jets using $\mathrm{R}=0.4$. The $60-80 \%$ centrality interval is used to define the peripheral reference. The most central collisions $(0-10 \%)$ exhibit a factor of two suppression relative to peripheral collisions, that varies weakly with jet $E_{\mathrm{T}}$. At fixed $E_{\mathrm{T}}$ the $R_{\mathrm{CP}}$ decreases monotonically with centrality. The greatest suppression of the $\mathrm{R}=0.4$ jets is similar to that of the $\mathrm{R}=0.2$ jets.

In summary, ATLAS has directly observed the suppression of single inclusive jet yields. The measured $R_{\mathrm{CP}}$ for two jet definitions, $\mathrm{R}=0.2$ and $\mathrm{R}=0.4$, indicate a significant suppression of these high energy jets.
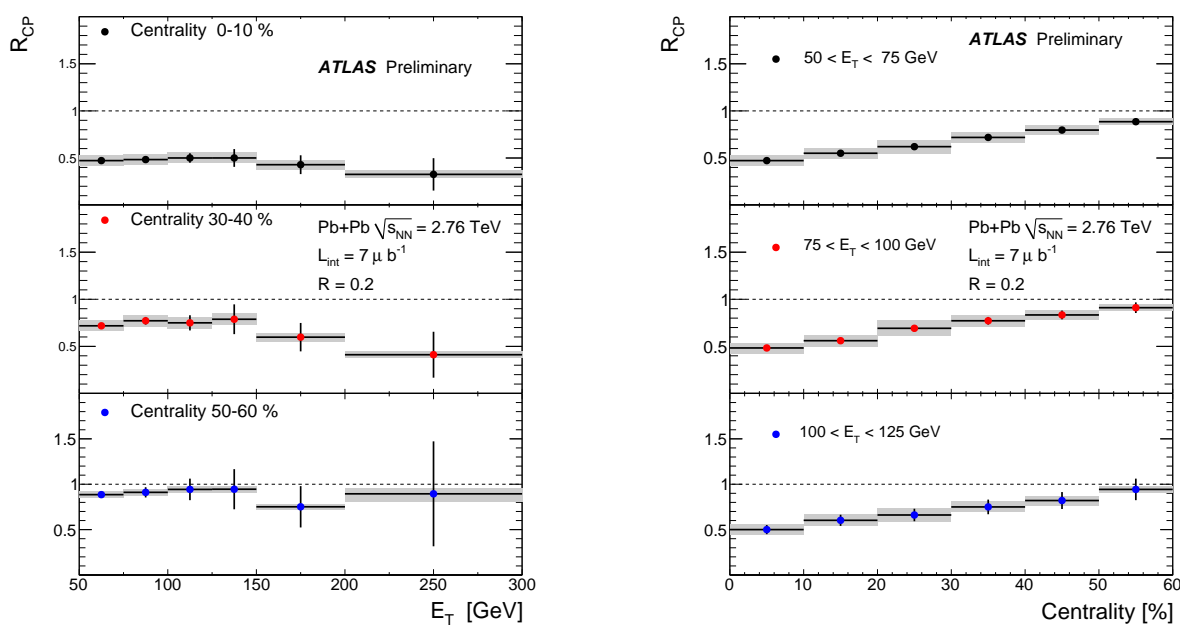

FIGURE 1. $R_{\mathrm{CP}}$ for $\mathrm{R}=0.2$ jets. Left: $R_{\mathrm{CP}}$ as a function of jet $E_{\mathrm{T}}$ for three centrality bins. Right: $R_{\mathrm{CP}}$ as a function of centrality for three $E_{\mathrm{T}}$ intervals. Error bars on the data points indicate statistical uncertainties, shaded errors represent combined systematic errors from jet energy resolution, jet energy scale variation with centrality and $N_{\text {coll }}$. 

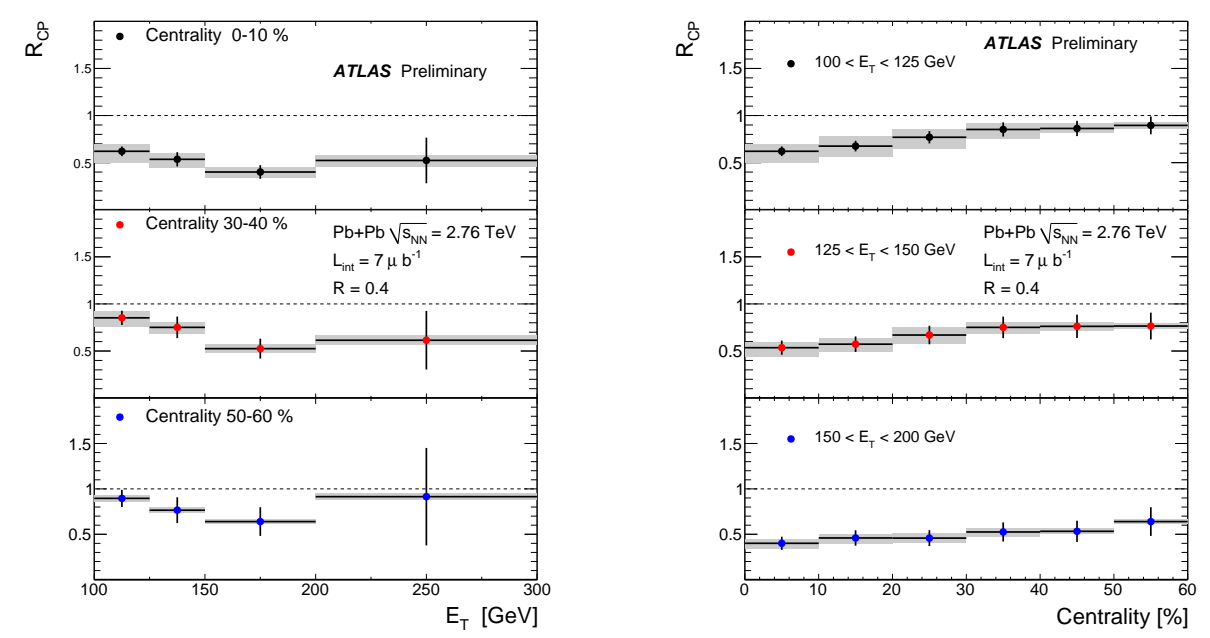

FIGURE 2. $R_{\mathrm{CP}}$ for $\mathrm{R}=0.4$ jets. Left: $R_{\mathrm{CP}}$ as a function of jet $E_{\mathrm{T}}$ for three centrality bins. Right: $R_{\mathrm{CP}}$ as a function of centrality for three $E_{\mathrm{T}}$ intervals. Error bars on the data points indicate statistical uncertainties, shaded errors represent combined systematic errors from jet energy resolution, jet energy scale variation with centrality and $N_{\text {coll }}$.

\section{REFERENCES}

1. A. Majumder, and M. Van Leeuwen (2010), arXiv:1002.2206 [hep-ph] .

2. K. Adcox, et al., Phys. Rev. Lett. 88, 022301 (2002).

3. C. Adler, et al., Phys. Rev. Lett. 89, 202301 (2002).

4. J. Bjorken (1982), fermilab-PUB-82/059-THY.

5. G. Aad, et al., Phys. Rev. Lett. 105, 252303 (2010).

6. I. Vitev, S. Wicks, and B.-W. Zhang, JHEP 11, 093 (2008).

7. ATLAS Collaboration, ATL-PHYS-PROC-2011-077, http://cdsweb.cern.ch/record/1366336.

8. ATLAS Collaboration, ATLAS-CONF-2011-075, http://cdsweb.cern.ch/record/1353220.

9. M. Cacciari, G. P. Salam, and G. Soyez, JHEP 04, 063 (2008).

10. ATLAS Collaboration, ATLAS-CONF-2010-053, http://cdsweb.cern.ch/record/1281310.

11. ATLAS Collaboration, ATLAS-CONF-2010-056, http://cdsweb.cern.ch/record/1281329. 